\title{
Range Extension Control of a Three-Wheel Electric Vehicle Prototype Based on Aggregation and Distribution
}

\author{
Binh-Minh Nguyen ${ }^{* a)}$ Member, \\ Michihiro Kawanishi* Member \\ Daichi Hasegawa \\ Non-member, \\ Kento Ohara* \\ Non-member \\ Tatsuo Narikiyo* Non-member
}

(Manuscript received Jan. 15, 2021, revised May 8, 2021)

J-STAGE Advance published date : June 11, 2021

\begin{abstract}
This paper presents an approach for the range extension control of a three-wheel electric vehicle prototype. By using the torque distribution vector to aggregate motor speeds, the physical model of the vehicle is mapped to an aggregation-and-distribution model (AaDM), which possesses the passivity property. Based on the AaDM, motion control and energy optimization can be designed separately. In particular, a speed controller was designed for the system to operate in the automatic cruise mode. A disturbance observer was designed to operate in the human driving mode. In this study, the conditions for the controllers were obtained to sufficiently ensure the $\mathrm{L}_{2}$ stability of the control system. The conditions can be checked conveniently without establishing the dynamical equation of the overall system. Under the practically reasonable assumption on motor parameters, the analytical solutions of the optimal torque distribution ratios and d-axis currents were approximately derived in this study. Various test scenarios were considered to validate the proposed control systems. The test results show that in either operation modes, the system can prevent wheel slip, thereby simultaneously improving motion control and energy minimization.
\end{abstract}

Keywords: electric vehicle, in-wheel-motor, range extension control system, passivity, disturbance observer, $\mathrm{L}_{2}$ stability

\section{Introduction}

1.1 RECS: A Successful Example of Motion Control

Thanks to the advances of electric motors, motion control of electric vehicle (EV) has grown up to be a mature research field. To mention just a few examples, we have antislip control ${ }^{(1)(2)}$, slip ratio control ${ }^{(3)(4)}$, and driving force control $^{(5)(6)}$. Recently, range extension control system (RECS) has been shown a great success ${ }^{(7)-(10)}$. The key idea of RECS is to find the driving distribution ratios that minimizes the summation of the output powers, the copper losses, and the iron losses ${ }^{(7)}$. The effectiveness of this idea has been verified through the history of RECS ${ }^{(7)-(10)}$. Literature review also shows several concepts which shared the common philosophy with RECS. For instance, Suzuki et al. developed a torque distribution algorithm for minimizing the tire dissipation energy ${ }^{(11)}$. Chatzikomis et al. proposed a torque vectoring algorithm for minimizing the power consumption of EVs driven by in-wheel-motors ${ }^{(12)}$. A hierarchically decentralized RECS was recently proposed by providing for each wheel a disturbance observer (DOB) based anti-slip control ${ }^{(13)}$. Despite of great successes already achieved, several issues still needed to be investigated.

1.2 Issues Raised from Practical Point of View First, it is well known that more energy would be required

a) Correspondence to: Binh-Minh Nguyen. E-mail: binhminh@ toyota-ti.ac.jp

* Department of Advanced Science and Technology, Control System Laboratory, Toyota Technological Institute

2-12-1, Hisakata, Tempaku-ku, Nagoya, Aichi 468-8511, Japan by the vehicle if wheel slip was not prevented. A straightforward idea is to design for each motor actuator a local controller $^{(1)-(4)(13)}$. However, this approach introduces the local wheel slip prevention command to the energy optimization problem. This increases the complexity of the optimization problem and challenges the attempt to obtain its analytical solutions. Therefore, how to independently design the energy optimization algorithm and motion control algorithm, especially slip prevention, is still an open issue.

Second, RECS ${ }^{(7)-(10)(13)}$ only dealt with driving torque allocation under the assumption such that the d-axis currents of the motors were neglectable. However, it is well known that motor loss can also be reduced via the d-axis components of armature current ${ }^{(14)}$. Hence, it is reasonable to address this current component in the general optimization problem of RECS. This issue has been preliminarily discussed in a study of RECS ${ }^{(8)}$ in which the authors showed that the problem can be solved numerically. Certainly, the discussion needs to be continued. Is it possible to derive for the optimization problem an approximated solution with analytical expression to reduce the computational burden of RECS?

Third, EV is actually a complex dynamical system with both nonlinearities and uncertainties. The nonlinearities exist due to the tire force characteristics ${ }^{(15)}$. The main uncertainties exist due to the change of road condition. Thus, system stabilization is a nontrivial issue for not only RECS but also other vehicle motion controls. An attempt to deal with RECS stabilization was made by treating the system as four independent wheel speed control loops ${ }^{(9)}$. As shown recently, the physical interaction between motor actuators actually exist and must 
be considered to guarantee system stability ${ }^{(16)(17)}$. However, linearization of the vehicle dynamics was required for applying the "linear quadratic regulator" ${ }^{(16)}$ and the "generalized frequency variable" ${ }^{(17)}$. This increased the complexity of system design. Hence, the following question raises naturally: How to stabilize RECS by a practical design procedure?

1.3 Problem Setting and Contributions RECS has been developed for a class of four-IWM-EV ${ }^{(7)-(10)}$. To extend the application range of RECS, this paper considered a threewheel EV prototype with two front IWMs and a rear motor and gear mechanism, all the motors are permanent magnet synchronous motors (PMSMs).

Using passivity theory, this paper shows that the vehicle is a passive system from the motor torques to the motor speeds. By a pair of torque distribution and speed aggregation vectors, the physical model of the vehicle can be mapped to a single-input single output model which also possesses passivity notation. This model is named aggregationand-distribution model (AaDM). Thanks to the passivity of AaDM, energy optimization and motion controller can be designed separately. The optimization law is only to update the distribution and aggregation vectors. Besides, motion control is designed directly for the AaDM.

This paper proposed two RECSs for automatic cruise mode (ACM) and human driving mode (HDM), respectively. Thanks to its simplicity and efficacy, DOB ${ }^{(1)(18)}$ was intentionally selected for the HDM. Note that, the traditional way of DOB design treated the vehicle as a linear plant with normbounded perturbation $\Delta$. However, as discussed by Bickel et al. ${ }^{(19)}$ and Sariyildiz et al. ${ }^{(20)}$, this approach does not show system stability rigorously. Based on passivity theory, this paper presents the conditions for the controllers to sufficiently guarantee $\mathrm{L}_{2}$ stability of RECS in both operation modes. The conditions do not require linearization, and can be checked easily via linear matrix inequality without establishing the dynamical equation of the overall system.

Moreover, the optimization problem was formulated by both the distribution ratios and d-axis currents. In particular, this paper shows that, under practically reasonable assumption on motor parameters, it is possible to (approximately) derive the analytical solution of the optimization problem.

1.4 The Rest of This Paper Section II described the model of the vehicle under study. Section III introduced the fundamentals of passivity theory and analyzed the passivity properties of the vehicle. Section IV presented the general design procedure of RECS for ACM and HDM. Section V presented the energy optimization problem, and under reasonable assumption obtained for it an analytical solution. The proposed approach was evaluated in Section VI through various test scenarios. The conclusions and future study were stated in Section VII.

\section{Vehicle Model}

Fig. 1 describes the model of the three-wheel EV. The vehicle mass is denoted by $m$ and the wheel radius is $r$. The driving force and vertical force of wheel are $F_{i}$ and $Z_{i}$, respectively. The torque and rotational speed of the motor are denoted as $T_{m, i}$ and $\omega_{m, i}$. As described in Fig. 2, the rear wheel is driven via the gear with the ratio $N_{3}$. Assuming that the gear efficiency is $100 \%$, the following relationship holds for

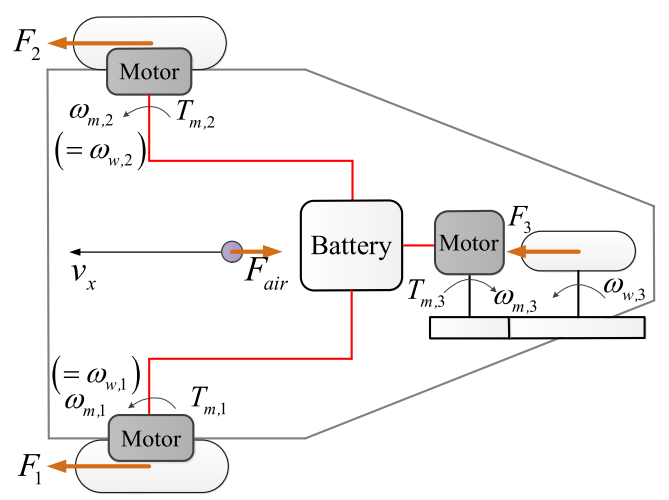

Fig. 1. Three-wheel EV model
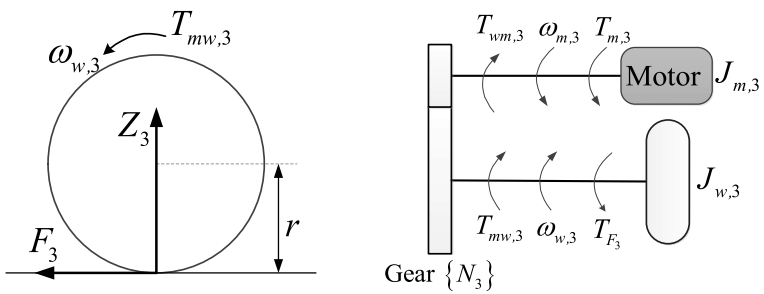

Fig. 2. Rotational motion of rear wheel

the rear wheel

$$
\omega_{m, 3}=N_{3} \omega_{w, 3}, \quad T_{w m, 3} \omega_{m, 3}=T_{m w, 3} \omega_{w, 3} \ldots \ldots
$$

Let the inertias of motor and wheel be $J_{m, 3}$ and $J_{w, 3}$, the rotational motion of the rear wheel and rear motor are

$$
\begin{aligned}
& J_{w, 3} \dot{\omega}_{w, 3}=T_{m w, 3}-T_{F_{3}}=T_{m w, 3}-r F_{3} \ldots \ldots \ldots \ldots \\
& J_{m, 3} \dot{\omega}_{m, 3}=T_{m, 3}-T_{w m, 3} \cdots \ldots \ldots \ldots \ldots \ldots \ldots \ldots
\end{aligned}
$$

Summarizing (1)-(3), the equivalent equation is expressed as

$$
J_{e q, 3} \dot{\omega}_{m, 3}=T_{m, 3}-N_{3}^{-1} r F_{3} \ldots \ldots \ldots \ldots \ldots \ldots \ldots
$$

where the equivalent inertia is

$$
J_{e q, 3}=J_{m, 3}+N_{3}^{-2} J_{w, 3}
$$

Let the inertia of the front wheel and motor be $J_{e q, i}(i=1$, 2 ). Define $N_{1,2}=1$, the rotational motion of the front motor actuators can be expressed similar to that of the rear wheel

$$
J_{e q, i} \dot{\omega}_{m, i}=T_{m, i}-N_{i}^{-1} r F_{i}, \quad i=1,2 \ldots \ldots \ldots \ldots
$$

The slip ratio of each wheel can be uniquely expressed as

$$
\lambda_{i}=\frac{r \omega_{w, i}-v_{x}}{\max \left\{r \omega_{w, i}, v_{x}, \varepsilon\right\}}
$$

where $v_{x}$ is the speed of the vehicle; $\varepsilon$ is a small positive number to prevent division by zero, and $\omega_{w, i} \triangleq \omega_{m, i} / N_{i}$ with $i=$ $1,2,3$. The nonlinear relationship between the driving force and the slip ratio (Fig. 3) is commonly described by "magic formula" ${ }^{(15)}$ :

$$
F_{i}=\left\{\begin{array}{ll}
f_{i}\left(\lambda_{i}\right) & \text { if } \lambda_{i} \geq 0 \\
-f_{i}\left(-\lambda_{i}\right) & \text { if } \lambda_{i}<0
\end{array} .\right.
$$

where 


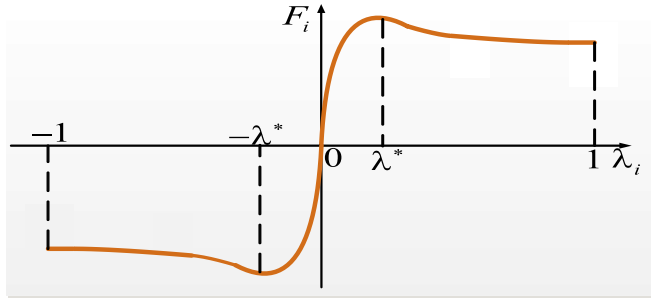

Fig. 3. Relationship between driving force and slip ratio

$$
f_{i}\left(\lambda_{i}\right)=A_{i} \sin \left\{B_{i} \tan ^{-1}\left[C_{i} \lambda_{i}-D_{i}\left(C_{i} \lambda_{i}-\tan ^{-1}\left(C_{i} \lambda_{i}\right)\right)\right]\right\}
$$

where $A_{i}=\mu_{i} Z_{i}, \mu_{i}$ is the friction coefficient; and $B_{i}, C_{i}$ and $D_{i}$ are the shape factors. Finally, the longitudinal motion of the vehicle is expressed as

$$
m \dot{v}_{x}=\sum_{i=1}^{3} F_{i}-\frac{1}{2} \rho C_{D} A_{F} v_{x}\left|v_{x}\right| \cdot
$$

where $\rho$ is air density, $C_{D}$ is the aerodynamical drag coefficient, and $A_{F}$ is the frontal area of the vehicle.

\section{Passivity Analysis of the Three-wheel EV}

3.1 Introduction to Passivity

$H$ given by the state space equation

$$
\left\{\begin{array}{l}
\dot{x}=f(x, u) \\
y=h(x, u)
\end{array}\right.
$$

where $u \in \mathbb{R}^{p}, y \in \mathbb{R}^{p}$, and $x \in \mathbb{R}^{n}$.

Definition $1^{(21)}$ : The system $H$ is passive from input $u$ to output $y$ if there exists a positive semidefinite function $S$ : $\mathbb{R}^{n} \rightarrow \mathbb{R}_{+}$, called storage function, such that $\dot{S} \leq y^{\dagger} u \forall x \in \mathbb{R}^{n}$, $u \in \mathbb{R}^{p}$. In addition, $H$ is input strictly passive (ISP) if

$$
\dot{S} \leq y^{\dagger} u-\delta_{u}\|u\|^{2} \forall x \in \mathbb{R}^{n}, u \in \mathbb{R}^{p} \text { for some } \delta_{u}>0,
$$

and output strictly passive (OSP) if

$$
\dot{S} \leq y^{\dagger} u-\delta_{y}\|y\|^{2} \forall x \in \mathbb{R}^{n}, u \in \mathbb{R}^{p} \text { for some } \delta_{y}>0 .
$$

Fig. 4 describes the system $H$ which is the feedback connection of $H_{1}$ and $H_{2}$. By Definition 1 , if both $H_{1}$ and $H_{2}$ are passive, then system $H$ with input $\left(\xi_{1}, \xi_{2}\right)$ and output $\left(y_{1}, y_{2}\right)$ is also passive. In addition, when $\xi_{2}=0$, system $H$ with input $\xi_{1}$ and output $y_{1}$ is shown to be passive. Moreover, passivity notation is related to a type of input-output stability, namely, $\mathrm{L}_{2}$-stability.

Theorem $2^{(21)}$ : If both $H_{1}$ and $H_{2}$ are OSP, then system $H$ with input $\left(\xi_{1}, \xi_{2}\right)$ and output $\left(y_{1}, y_{2}\right)$ has a finite $\mathrm{L}_{2}$-gain. When $\xi_{2}=0$, the system $H$ with input $\xi_{1}$ and output $y_{1}$ has a finite $\mathrm{L}_{2}$-gain if either (i) $H_{1}$ is passive and $H_{2}$ is ISP, or (ii) $H_{1}$ is OSP and $H_{2}$ is passive.

3.2 Passivity Analysis Based on (1)-(10), the vehicle is described by the $\mathbf{E V}$ system with the input and the output

$$
\mathbf{T}_{m}=\left[\begin{array}{lll}
T_{m, 1} & T_{m, 2} & T_{m, 3}
\end{array}\right]^{\dagger}, \quad \boldsymbol{\omega}_{m}=\left[\begin{array}{lll}
\omega_{m, 1} & \omega_{m, 2} & \omega_{m, 3}
\end{array}\right]^{\dagger}
$$

where $\dagger$ is the transpose notation.

Proposition 3: EV is passive with the storage function

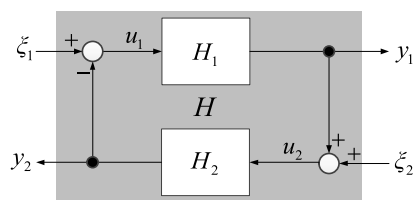

Fig. 4. Feedback connection of two sub-systems

$$
S=S_{V}+\sum_{i=1}^{3} S_{W, i} \text { where } S_{V}=\frac{1}{2} m v_{x}^{2}, S_{W, i}=\frac{1}{2} J_{e q, i} \omega_{m, i}^{2}
$$

Proof: From (4), (6), (10) and (13), we have

$$
\begin{aligned}
& \dot{S}_{V}=m \dot{v}_{x} v_{x}=\sum_{i=1}^{3} F_{i} v_{x}-\frac{1}{2} \rho C_{D} A_{F} v_{x}^{2}\left|v_{x}\right| \cdots \cdots \\
& \dot{S}_{W, i}=J_{e q, i} \dot{\omega}_{m, i} \omega_{m, i}=\omega_{m, i} T_{m, i}-N_{i}^{-1} r \omega_{m, i} F_{i} \cdots
\end{aligned}
$$

From (7), (8), (14) and (15), we have

$$
\begin{aligned}
\dot{S}= & \omega_{m}^{\dagger} \mathbf{T}_{m}-\sum_{i=1}^{3} \max \left\{r \omega_{w, i}, v_{x}, \varepsilon\right\} \lambda_{i} f_{i}\left(\lambda_{i}\right) \\
& -\frac{1}{2} \rho C_{D} A_{F} v_{x}^{2}\left|v_{x}\right| \ldots \ldots \ldots \ldots \ldots \ldots \ldots
\end{aligned}
$$

As can be seen from the tire force characteristics in Fig. 3, the term $\lambda_{i} f_{i}\left(\lambda_{i}\right)$ is always non-negative. Consequently, it can be shown from (16) that the following inequality holds true, which also shows that the system EV is passive by Definition 1:

$$
\dot{S} \leq \omega_{m}^{\dagger} \mathbf{T}_{m}
$$

\section{General Design of RECS}

4.1 Aggregation-and-distribution Model The proposed aggregation-and-distribution model (AaDM) is described in Fig. 5 with the scalar input $T_{a}$ and the scalar output $\omega_{a}$. The idea of the model is explained as follows. Since the front wheels are directly driven by in-wheel-motors, $\omega_{m, 1}$ and $\omega_{m, 2}$ also represent the rotational speeds of the front wheels. However, the rear wheel is not directly connected to the motor, but via a gear mechanism with the ratio $N_{3}$. As shown in (1), the rotational speed of the rear wheel $N_{3}$ is smaller than that of the rear motor. Therefore, the motor speeds should be scaled down before aggregation. By applying a pair of scaling matrix $\operatorname{diag}\left\{N_{i}^{-1}\right\}$ to $\mathbf{E V}$, we obtain in Fig. 5 a system $\mathbf{E V}_{C}$ with the input and the output

$$
\begin{aligned}
& \boldsymbol{\omega}_{c}=\operatorname{diag}\left\{N_{i}^{-1}\right\} \boldsymbol{\omega}_{m}=\left[\begin{array}{lll}
\omega_{m, 1} & \omega_{m, 2} & N_{3}^{-1} \omega_{m, 3}
\end{array}\right]^{\dagger} \cdots \\
& \mathbf{T}_{m}=\operatorname{diag}\left\{N_{i}^{-1}\right\} \mathbf{T}_{c}=\left[\begin{array}{lll}
T_{c, 1} & T_{c, 2} & N_{3}^{-1} T_{c, 3}
\end{array}\right]^{\dagger} \cdots \cdots
\end{aligned}
$$

where $\mathbf{T}_{c}$ is distributed from $T_{a}$, and $\omega_{c}$ is aggregated to be $\omega_{a}$. Considering only the longitudinal motion of the vehicle, the aggregation/distribution vector is defined as

$$
\mathbf{k}=\left[\begin{array}{lll}
k_{1} & k_{2} & k_{3}
\end{array}\right]^{\dagger}
$$

where the distribution ratios satisfy the following constraints

$$
\left\{\begin{array}{l}
k_{1}=k_{2}, k_{1}+k_{2}+k_{3}=1 \\
0 \leq k_{1,2,3} \leq 1
\end{array}\right.
$$




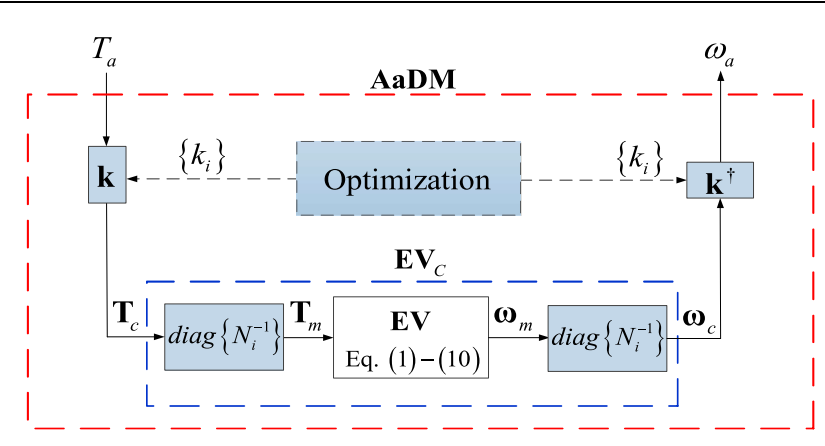

Fig. 5. Block diagram of AaDM

With respect to (17), (18) and (19), we have

$$
\boldsymbol{\omega}_{c}^{\dagger} \mathbf{T}_{c}=\left[\operatorname{diag}\left\{N_{i}^{-1}\right\} \boldsymbol{\omega}_{m}\right]^{\dagger} \mathbf{T}_{c}=\boldsymbol{\omega}_{m}^{\dagger} \mathbf{T}_{m} \geq \dot{S} \ldots \ldots
$$

which means $\mathbf{E} \mathbf{V}_{C}$ is also passive with the storage function (13). Hence, the scaling matrix $\operatorname{diag}\left\{N_{i}^{-1}\right\}$ has three meanings. First, it is a map to transform motor speed to wheel speed. Second, it is a map to transform wheel driving torque to motor torque command. Last but not least, it maintains the passivity property of the vehicle.

With respect to $(21), \omega_{a}$ is almost close to the rotational speed of any single wheel if the slip ratio is small. In the control space, we can treat the AaDM as a "virtual EV" with the input torque $T_{a}$ and the wheel speed $\omega_{a}$. By applying motion control to AaDM, it is possible to separate the motion control algorithm and energy optimization algorithm which is designed for the inner of AaDM.

Proposition 4: AaDM is passive with the storage function (13).

Proof: From (22) and the structure of AaDM, we have

$$
\omega_{a}^{\dagger} T_{a}=\left(\mathbf{k}^{\dagger} \omega_{c}\right)^{\dagger} T_{a}=\omega_{c}^{\dagger}\left(\mathbf{k} T_{a}\right)=\omega_{c}^{\dagger} \mathbf{T}_{c} \geq \dot{S} \cdots
$$

which shows the passivity of AaDM.

Note that, the passivity notation of AaDM holds even when the distribution ratios are time-varying. This motivates us to consider the following design approach of RECS.

4.2 ACM-RECS The control system for the ACM was presented in Fig. 6 in which the reference speed is represented by a scalar signal $\omega_{r}$. Its design procedure is presented as follows.

\section{ACM-RECS design procedure}

Stage 1: Establish the algorithm to find the distribution ratios and the d-axis currents that minimize be the summation of motor output powers, copper loss, and iron loss. The optimization problem is solved under the constraint (21).

Stage 2: Design the controller $C_{a}(s)$ which is OSP.

Proposition 5: The ACM-RECS design procedure sufficiently guarantees that the overall system in Fig. 6 with input $\omega_{r}$ and output $T_{a}$ has a finite $\mathrm{L}_{2}$ gain.

Proof: The system in Fig. 6 is actually the feedback connection of the passive system AaDM and $C_{a}(s)$. According to Theorem 2, if $C_{a}(s)$ is OSP then the system (with input $\omega_{r}$ and output $T_{a}$ ) has a finite $\mathrm{L}_{2}$ gain and is $\mathrm{L}_{2}$ stable. This completes the proof.

4.3 HDM-RECS The control system for HDM was described in Fig. 7(a) in which the DOB is established with the nominal model $P_{n}(s)$ and the low-pass filter $Q(s) . T_{a}$ is the summation of the driving command given by the

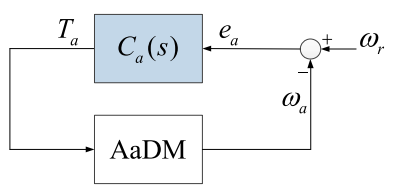

Fig. 6. Block diagram of ACM-RECS

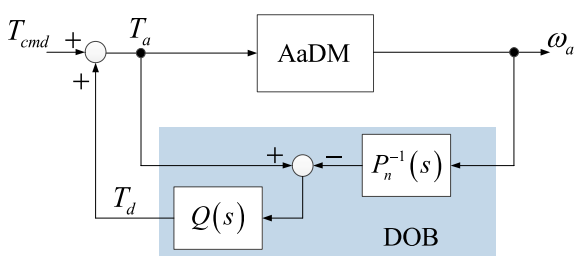

(a) Original block diagram.

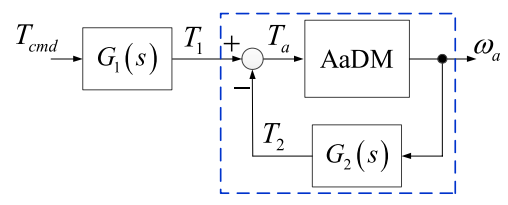

(b) Equivalent expression.

Fig. 7. Block diagram of HDM-RECS

driver $\left(T_{c m d}\right)$ and the anti-slip command given by DOB $\left(T_{d}\right)$. Fig. 7(b) describes the equivalent block diagram of HDMRECS with

$$
G_{1}(s)=\frac{1}{1-Q(s)}, \quad G_{2}(s)=\frac{Q(s) P_{n}^{-1}(s)}{1-Q(s)} \cdots \cdots
$$

\section{HDM-RECS design procedure}

Stage 1: Establish the algorithm to find the distribution ratios and the d-axis currents that minimize be the summation of motor output powers, copper loss, and iron loss. The optimization problem is solved under the constraints (21).

Stage 2: Select the nominal model and the low pass filter such that $G_{1}(s)$ is a stable transfer function and $G_{2}(s)$ is ISP. Proposition 6: The HDM-RECS design procedure sufficiently guarantees that the feedback connection in Fig. 7(b) with input $T_{1}$ and output $\omega_{a}$ has a finite $\mathrm{L}_{2}$ gain.

Proof: The stability of $G_{1}(s)$ is to ensures the internal stability from $T_{c m d}$ to $T_{1}$. As AaDM is passive, if $G_{2}(s)$ is ISP then the system in the green-dashed rectangle of Fig. 7(b) has a finite $\mathrm{L}_{2}$ gain and is $\mathrm{L}_{2}$ stable. This completes the proof.

As shown in Fig. 7, instead of designing for each motor a DOB, we only design a DOB for AaDM. In the Section 6, we will show that this control configuration is enough to prevent the wheel from the slip phenomenon. This control configuration has another merit in comparison with the decentralized DOB $^{(13)}$ : The optimization problem was not intervened by the DOB command. This allows us to separate the motion control and optimization algorithms. Hence, ACM-RECS and HDM-RECS can share the same optimization algorithm designed insight AaDM.

4.4 Remark Several points should be noticed when applying the proposed design approach.

Remark 1: If the gear efficiency $\eta_{3}$ should be addressed, the $\mathbf{E V}$ system is still passive from $\mathbf{T}_{m}$ to the output $\operatorname{diag}\left\{1,1, \eta_{3}\right\} \omega_{m}$ with the storage function $\mathrm{S}=S_{V}+S_{W, 1}+$ $S_{W, 2}+\eta_{3} S_{W, 3}$. Hence, the proposed approach is still applicable without special difficulty. 
Remark 2: If the dead-time of the motor driver is large enough, and the saturation of the motor torque/current should be addressed, then EV and AaDM are no longer passive systems. Fortunately, we might benefit from other passivity properties of the vehicle. For instance, the map from the slip ratio to the driving force is passive since $\lambda_{i} f_{i}\left(\lambda_{i}\right)$ is nonnegative. Thus, it is possible to lump the nonlinear map and the torque saturation operator into a memoryless map with sector-bounded. This allows us to further examine the "absolute stability" of RECS by using Circle-Popov criteria ${ }^{(21)}$.

\section{Optimization Problem}

5.1 Derivation of the Optimization Problem Given a torque command $T_{a}$ and a vehicle speed $v_{x}$, HDM-RECS and ACM-RECS share the same optimization problem which will be examined in this Section. Since $\mathrm{L}_{2}$ stability is ensured by the proposed design procedures, it is possible to assume that the slip ratio is maintained at small value. The driving force can be linearized as $F_{i}=D_{s, i} Z_{i} \lambda_{i}$ where $D_{s, i}$ is the driving stiffness coefficient. The motor speed can be approximated as ${ }^{(8)}$ :

$$
\omega_{m, i} \approx \frac{N_{i} v_{x}}{r}\left(1+\lambda_{i}\right) \approx \frac{N_{i} v_{x}}{r}\left(1+\frac{F_{i}}{D_{s, i} Z_{i}}\right) \cdots \cdots \cdots
$$

The motor torque and the driving force can be expressed as

$$
\begin{gathered}
T_{m, i}=\frac{k_{i} T_{a}}{N_{i}} \ldots \ldots \ldots \ldots \ldots \ldots \\
F_{i} \approx \frac{N_{i}}{r} T_{m, i} \approx \frac{N_{i}}{r} \frac{k_{i} T_{a}}{N_{i}}=\frac{k_{i} T_{a}}{r}
\end{gathered}
$$

From (25)-(27), the output power $P_{M, i}$ is derived as in Table 1. Next, this paper examines in Fig. 8 the equivalent circuit of PM motor ${ }^{(14)}$. In this figure, the $\mathrm{d}$ - and q-axis components of armature current are $I_{d, i}$ and $I_{q, i}$, respectively. $I_{c d, i}$ and $I_{c q, i}$ represent the d- and q-axis components of iron loss current, respectively. The $\mathrm{d}$ - and q-axis components of the terminal voltage are denoted as $U_{d, i}$ and $U_{q, i}$, respectively. The armature winding resistance per-phase and the iron loss resistance are $R_{a, i}$ and $R_{c, i}$, respectively. $L_{d, i}$ and $L_{q, i}$ are the dand q-axis components of armature self-inductance, respectively. Let $\Psi_{e, i}$ be the flux linkage of permanent magnet perphase, we define

$$
\Psi_{a, i}=\sqrt{3} \Psi_{e, i}
$$

As shown in (14), we have

$$
\begin{gathered}
I_{o d, i}=I_{d, i}-I_{c d, i}, \quad I_{o q, i}=I_{q, i}-I_{c q, i} \ldots \ldots \cdots \cdots \\
I_{c d, i}=-\frac{\omega_{e, i} \rho_{i} L_{d, i} I_{o q, i}}{R_{C, i}}, \quad I_{c q, i}=\frac{\omega_{e, i}\left(\Psi_{a, i}+L_{d, i} I_{o d, i}\right)}{R_{C, i}} \\
\ldots \ldots \ldots \ldots \cdots \cdots \cdots \cdots \\
\omega_{e, i}=p_{n, i} \omega_{m, i} \approx \frac{p_{n, i} N_{i} v_{x}}{r} \cdots \cdots \cdots \cdots \cdots
\end{gathered}
$$

where $p_{n, i}$ is the number of pole pairs. Define $\rho_{i} \triangleq L_{q, i} / L_{d, i}$ as the salient coefficient, the motor torque is expressed as

$$
\begin{aligned}
& T_{m, i}=p_{n, i} \Psi_{e q, i} I_{o q, i} \cdots \ldots \ldots \\
& \Psi_{e q, i}=\Psi_{a, i}+\left(1-\rho_{i}\right) L_{d, i} I_{o d, i}
\end{aligned}
$$

The copper loss and the iron loss are
Table 1. Derivation of motor powers

\begin{tabular}{|c|c|}
\hline Power & Formula \\
\hline Output & $P_{M, i}=\frac{v_{x}}{r}\left(1+\frac{k_{i} T_{a}}{r D_{s, i} Z_{i}}\right) k_{i} T_{a}$ \\
\hline $\begin{array}{c}\text { Copper } \\
\text { loss }\end{array}$ & $P_{C u, i}=R_{a, i}\left\{\left(I_{o d, i}-\frac{v_{x} \rho_{i} L_{d, i}}{r R_{c, i}} \frac{k_{i} T_{a}}{\Psi_{e q, i}}\right)^{2}\right.$ \\
$\left.+\left[\frac{1}{p_{n, i} \Psi_{e q, i}} \cdot \frac{k_{i} T_{a}}{N_{i}}+\frac{p_{n, i} N_{i} v_{x}\left(\Psi_{a, i}+L_{d, i} I_{o d, i}\right)}{r R_{c, i}}\right)^{2}\right\}$ \\
\hline Iron loss & $P_{F e, i}=\frac{p_{n, i}^{2} N_{i}^{2} v_{x}^{2}}{r^{2} R_{c, i}}\left\{\frac{\rho_{i}^{2} L_{d, i}^{2} k_{i}^{2} T_{a}^{2}}{p_{n, i}^{2} \Psi_{e q, i}^{2} N_{i}^{2}}+\left(\Psi_{a, i}+L_{d, i} I_{o d, i}\right)^{2}\right\}$ \\
\hline
\end{tabular}
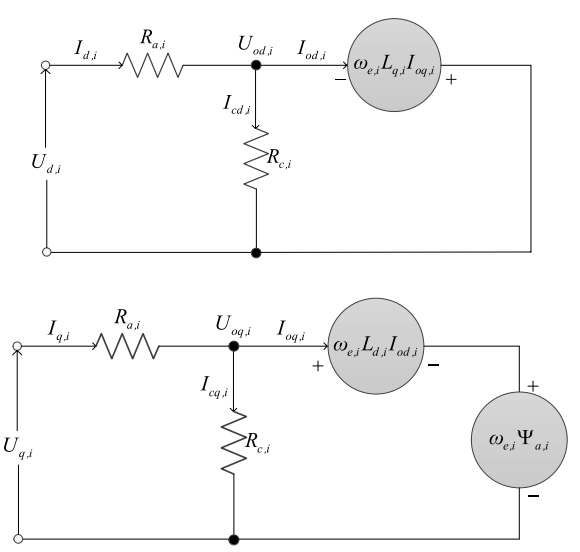

Fig. 8. Equivalent circuit of the PM motor

$$
P_{C u, i}=R_{a, i}\left(I_{d, i}^{2}+I_{q, i}^{2}\right), \quad P_{F e, i}=R_{c, i}\left(I_{c d, i}^{2}+I_{c q, i}^{2}\right)
$$

Substitute (26) and (29)-(32) into (34), the copper loss and iron loss are finally expressed as in Table 1 . In this study, the inverter loss and other power losses are assumed to be uncontrollable. We can define the summation of the powers in Table 1 as

$$
P_{\text {in }}=\sum_{i=1}^{3}\left(P_{M, i}+P_{C u, i}+P_{F e, i}\right) \cdots \ldots \ldots \ldots \ldots \ldots \ldots \ldots
$$

Note that, $P_{C u, i}$ and $P_{F e, i}$ are the convex functions of both $k_{i}$ and $I_{o d, i}$, and $P_{M, i}$ is a convex function of $k_{i}$. Therefore, the following optimization problem (OP) can be considered for RECS:

$$
\begin{array}{r}
\text { OP: } \min _{\left\{k_{i}, I_{\text {od, },}\right\}} P_{\text {in }} \\
\text { s.t. }(21) \cdots
\end{array}
$$

The OP (36) is three-wheel version of the optimization problem discussed by Fujimoto et al. ${ }^{(8)}$ when considering an EV driven by four IWMs. In (21), we can let

$$
k_{1}=k_{2}=k_{f}, k_{3}=1-2 k_{f} \quad \text { where } 0 \leq \mathrm{k}_{f} \leq 0.5
$$

Substituting (37) into (36), it can be shown that

$$
P_{\text {in }}=P_{\text {in }}\left(k_{f}, I_{o d, 1}, I_{o d, 2}, I_{o d, 3}\right)
$$


Table 2. Practical algorithm for the energy optimization problem of RECS

\begin{tabular}{|c|c|c|}
\hline Step & Purpose & Detail algorithm \\
\hline \multirow{2}{*}{1} & \multirow{2}{*}{$\begin{array}{l}\text { Update the values of } \\
\alpha_{n, i}, \beta_{i} \text { and } \gamma_{i} \text { using } \\
\text { the estimated values of } \\
\text { vertical forces, driving } \\
\text { stiffness, and a speed } v_{x}\end{array}$} & $\alpha_{n, i}=R_{a, i}\left[\left(\frac{v_{x} \rho_{i} L_{d, i}}{r R_{c, i} \Psi_{a, i}}\right)^{2}+\left(\frac{1}{N_{i} p_{n, i} \Psi_{a, i}}\right)^{2}\right]+\frac{v_{x}^{2} \rho_{i}^{2} L_{d, i}^{2}}{r^{2} R_{c, i} \Psi_{a, i}^{2}}+\frac{v_{x}}{r^{2} D_{s, i} Z_{i}}$ \\
\hline & & $\beta_{i}=R_{a, i}+\left(R_{a, i}+R_{c, i}\right)\left(\frac{p_{n, i} N_{i} v_{x}}{r R_{c, i}}\right)^{2} L_{d, i}^{2}, \quad \gamma_{i}=2\left(R_{a, i}+R_{c, i}\right)\left(\frac{p_{n, i} N_{i} v_{x}}{r R_{c, i}}\right)^{2} \Psi_{a, i} L_{d, i}$ \\
\hline 2 & $\begin{array}{l}\text { Obtain the optimal value } \\
\text { of } I_{o d, i} \text { and } k_{k}\end{array}$ & $I_{o d, i}^{*}=-\frac{\gamma_{i}}{2 \beta_{i}}, k_{f}^{*}=\frac{2 \alpha_{n, 3}}{\alpha_{n, 1}+\alpha_{n, 2}+4 \alpha_{n, 3}}$ \\
\hline \multirow[b]{2}{*}{3} & \multirow{2}{*}{$\begin{array}{l}\text { Update the distribution } \\
\text { ratios and the d- and q- } \\
\text { axis currents }\end{array}$} & $k_{1}=k_{2}=k_{f}^{*}, k_{3}=1-2 k_{f}^{*}$ \\
\hline & & $I_{d, i}=I_{o d, i}^{*}-\frac{v_{x}}{r} \frac{\rho_{i} L_{d, i}}{R_{C, i}} \frac{k_{i} T_{a}}{\left[\Psi_{a, i}+\left(1-\rho_{i}\right) L_{d, i} I_{o d, i}\right]}, I_{q, i}=\frac{k_{i} T_{a}}{p_{n, i} N_{i}\left[\Psi_{a, i}+\left(1-\rho_{i}\right) L_{d, i} I_{o d, i}^{*}\right]}+\frac{p_{n, i} N_{i} v_{x}\left(\Psi_{a, i}+L_{d, i} I_{o d, i}^{*}\right)}{r R_{C, i}}$ \\
\hline
\end{tabular}

As discussed by Fujimoto et al. ${ }^{\left({ }^{8}\right)}$, the optimal values of the distribution ratios and the d-axis currents can be obtained by numerically solving a set of equations

$$
\frac{\partial P_{i n}}{\partial k_{f}}=0, \frac{\partial P_{i n}}{\partial I_{o d, 1}}=0, \frac{\partial P_{i n}}{\partial I_{o d, 2}}=0, \frac{\partial P_{i n}}{\partial I_{o d, 3}}=0 \cdots
$$

5.2 A Practical Solution of OP The numerical algorithm, however, will increase the computational burden. This paper aims to find an approximated solution of OP, which can be expressed analytically. Based on Table 1, we notice that $P_{\text {in }}$ can be represented as

$$
\begin{aligned}
& P_{\text {in }}=P^{U}+\sum_{i=1}^{3} P_{i}{ }^{L} \\
& P^{U}=\sum_{i=1}^{3} P_{i}^{U} \\
& P_{i}^{U}=\alpha_{i}\left(k_{i} T_{a}\right)^{2}+\frac{v_{x}}{r}\left(1+\frac{2 R_{a, i}}{R_{c, i}}\right) k_{i} T_{a} \\
& \alpha_{i}=R_{a, i}\left[\left(\frac{v_{x} \rho_{i} L_{d, i}}{r R_{c, i} \Psi_{e q, i}}\right)^{2}+\left(\frac{1}{N_{i} p_{n, i} \Psi_{e q, i}}\right)^{2}\right] \\
& +\frac{v_{x}^{2} \rho_{i}^{2} L_{d, i}^{2}}{r^{2} R_{c, i} \Psi_{e q, i}^{2}}+\frac{v_{x}}{r^{2} D_{s, i} Z_{i}} \\
& P_{i}{ }^{L}=\beta_{i} I_{o d, i}^{2}+\gamma_{i} I_{o d, i}+\left(R_{a, i}+R_{c, i}\right)\left(\frac{p_{n, i} N_{i} v_{x}}{r R_{c, i}}\right)^{2} \Psi_{a, i}^{2} \\
& \beta_{i}=R_{a, i}+\left(R_{a, i}+R_{c, i}\right)\left(\frac{p_{n, i} N_{i} v_{x}}{r R_{c, i}}\right)^{2} L_{d, i}^{2} \\
& \gamma_{i}=2\left(R_{a, i}+R_{c, i}\right)\left(\frac{p_{n, i} N_{i} v_{x}}{r R_{c, i}}\right)^{2} \Psi_{a, i} L_{d, i}
\end{aligned}
$$

Assumption: We assume that

$$
\begin{gathered}
\frac{2 R_{a, i}}{R_{c, i}} \ll 1, \quad \forall i \in\{1,2,3\} \\
\Psi_{e q, i} \approx \Psi_{a, i} \quad \forall i \in\{1,2,3\}
\end{gathered}
$$

The inequality (47) is practically reasonable. In many PMSM drives, we notice that $R_{a, i}$ is commonly small in comparison with $R_{c, i}$. For instance, the PMSM studied by Morimoto et al. ${ }^{(14)}$ has

$$
2 R_{a} / R_{c}=2 \times 0.57 / 240=0.0081
$$

From a practical point of view, we know that $L_{d, i} I_{o d, i}$ is relatively small in comparison with $\Psi_{a, i}$. Hence, (48) will hold almost surely if the salient coefficient is closed to 1 . In addition, $\Psi_{e q, i}=\Psi_{a, i}$ holds true if the motor has not saliency.

Under the above assumption, we can approximate

$$
P_{\text {in }} \approx \bar{P}_{\text {in }}=\bar{P}^{U}+\sum_{i=1}^{3} P_{i}{ }^{L} .
$$

With respect to (37), we have in (49)

$$
\begin{aligned}
\bar{P}^{U} & =T_{a}^{2}\left[\left(\alpha_{n, 1}+\alpha_{n, 2}+4 \alpha_{n, 3}\right) k_{f}^{2}-4 \alpha_{n, 3} k_{f}+\alpha_{n, 3}\right]+\frac{T_{a} v_{x}}{r} \\
\ldots \ldots \ldots \ldots \ldots \ldots \ldots & \ldots \ldots \ldots \ldots \ldots \ldots \\
\alpha_{n, i} & =R_{a, i}\left[\left(\frac{v_{x} \rho_{i} L_{d, i}}{r R_{c, i} \Psi_{a, i}}\right)^{2}+\left(\frac{1}{N_{i} p_{n, i} \Psi_{a, i}}\right)^{2}\right] \\
& +\frac{v_{x}^{2} \rho_{i}^{2} L_{d, i}^{2}}{r^{2} R_{c, i} \Psi_{a, i}^{2}}+\frac{v_{x}}{r^{2} D_{s, i} Z_{i}} \ldots \ldots \ldots \ldots \ldots \ldots
\end{aligned}
$$

We notice that, given a command $T_{a}$ and a speed $v_{x}$ :

- Each $P_{i}^{L}$ is a quadratic function of only $I_{o d, i}$.

- $\bar{P}^{U}$ is a quadratic function of only $k_{f}$.

Instead of solving (36) directly, we consider the minimization of the approximated function (49) by solving

$$
\frac{\partial \bar{P}_{i n}}{\partial k_{f}}=0, \frac{\partial \bar{P}_{i n}}{\partial I_{o d, 1}}=0, \frac{\partial \bar{P}_{i n}}{\partial I_{o d, 2}}=0, \frac{\partial \bar{P}_{i n}}{\partial I_{o d, 3}}=0 \cdots \cdots
$$

In summary, a practical algorithm for obtaining an approximated solution of the energy optimization problem was shown in Table 2. As can be seen from (51), each $\alpha_{n, i}$ is a positive number. Hence, the distribution ratios calculated in Table 2 will certainly satisfy the condition (21). With respect to (29), (30) and (31), the d- and q-axis currents are finally calculated as in Table 2.

Notice that, the driving stiffness can be estimated easily using the motor torque and motor speed ${ }^{(5)}$. Also, the vertical force can be calculated using on-board sensors ${ }^{(8)}$. Since this paper is to demonstrate the idea of aggregation-distribution for RECS, we did not present such estimation algorithms which can be implemented in real-time without special difficulty. 


\section{Evaluation and Discussion}

6.1 Vehicle Model Three-wheel vehicles have been developed from the beginning of automotive history. They have been used for various purposes, such as personal transportation and racing. For instance, Toyota has developed the concept of i-ROAD EV with two front wheels. In this study, we demonstrated the proposed approach by a three-wheel recreational EV prototype ${ }^{(22)}$. The main physical parameters of the vehicle are presented in app. Table 1 of the Appendix.

6.2 Control System Design First, we presented the condition for selecting the controller $C_{a}(s)$ of the ACMRECS. According to Proposition 5, $C_{a}(s)$ should be OSP. This paper considered the class of compensator

$$
C_{a}(s)=K_{a} \frac{s+a_{1}}{s+a_{2}}
$$

where $K_{a}>0, a_{1}>a_{2}>0$. As shown in Fig. 6 , the above controller can be represented in state space as

$$
\left\{\begin{array}{l}
\dot{x}_{a}=-a_{2} x_{a}+b e_{a} \\
T_{a}=K_{a} x_{a}+K_{a} e_{a}
\end{array}\right.
$$

where $b=a_{1}-a_{2}$. Applying the Kalman-Yakubovich-Popov (KYP) Lemma ${ }^{(23)}$ to the system (54), $C_{a}(s)$ is OSP if there exist the positive numbers $\phi$ and $\delta$ such that the following matrix is negative semidefinite

$$
Q_{A C M}=\left[\begin{array}{cc}
-2 \phi a_{2}+2 \delta K_{a}^{2} & \phi b-K_{a}+2 \delta K_{a}^{2} \\
\phi b-K_{a}+2 \delta K_{a}^{2} & -2 K_{a}+2 \delta K_{a}^{2}
\end{array}\right] \leq 0
$$

The above condition can be checked without special difficulty by Matlab program. In practical application, the motor torque has its maximum value. Therefore, the gain $K_{a}$ has a certain upper-bound. If $K_{a}$ is bigger than this upper-bound, the system might suffer the fluctuation of the motor torque. Hence, the set of $\left\{K_{a}, a_{1}, a_{2}\right\}$ could be selected from a finetuning process with the condition (55).

Next, this paper considered the following nominal model and low pass filter for the DOB in HDM-RECS.

$$
P_{n}(s)=\frac{1}{J_{n} s+D_{n}}, \quad Q(s)=\frac{K_{f}}{\tau_{f} s+1} .
$$

where $P_{n}(s)$ represents the nominal dynamics of the AaDM. It consists of two parameters $J_{n}$ and $D_{n}$. On the other hand, $Q(s)$ has the time constant $\tau_{f}$ and $K_{f}$ is the tuning gain which should be between 0 and 1 . With the above setting, we have

$$
G_{1}(s)=\frac{\tau_{f} s+1}{\tau_{f} s+1-K_{f}}, \quad G_{2}(s)=K_{f} \frac{J_{n} s+D_{n}}{\tau_{f} s+1-K_{f}}
$$

which shows that $G_{1}(s)$ is stable for any positive time constant of the DOB, and all tuning gain selected between 0 and 1. Applying the KYP Lemma, $G_{2}(s)$ is ISP if there exist the positive numbers $\chi$ and $\beta$ such that

$$
Q_{H D M}=\left[\begin{array}{cc}
-2 \frac{1-K_{f}}{\tau_{f}} \chi & \xi \\
\xi & -2 \frac{K_{f} J_{n}}{\tau_{f}}+2 \beta
\end{array}\right] \leq 0 \cdots \cdots
$$

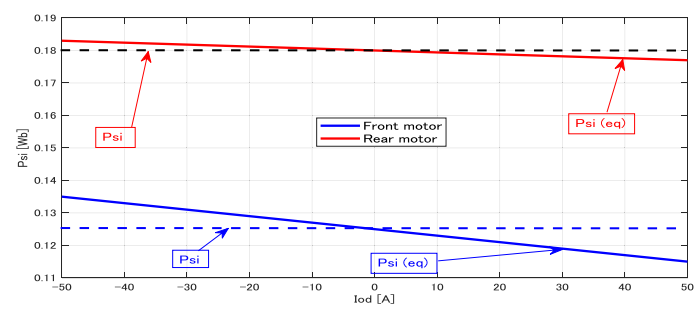

Fig. 9. Comparison of $\Psi_{e q, i}$ and $\Psi_{a, i}$ for the three-wheel EV

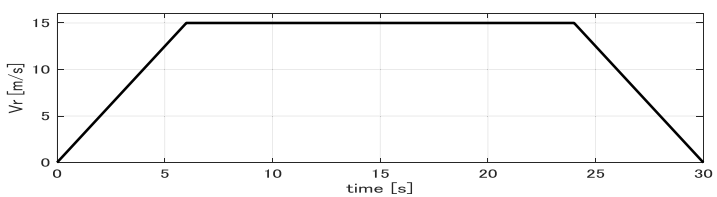

(a) Speed pattern

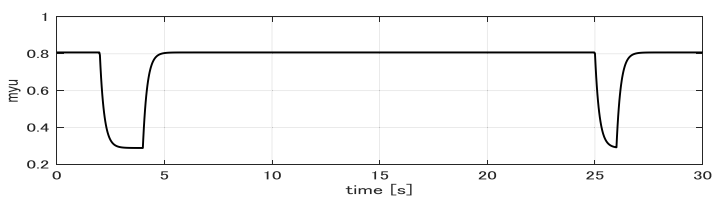

(b) Change of road friction.

Fig. 10. ACM-RECS: Simulation setting of Test 1

where

$$
\xi=\left(\frac{D_{n}}{\tau_{f}}-\frac{1-K_{f}}{\tau_{f}^{2}} J_{n}\right) \chi-K_{f} \ldots \ldots \ldots \ldots \ldots \ldots
$$

6.3 Evaluation of ACM-RECS By fine-tuning process with the condition (55), we selected the controller $C_{a}(s)$ with $K_{a}=295, a_{1}=21.2$, and $a_{2}=4.1$. We can verify that this controller is OSP. For instance, by selecting

$$
\phi=\frac{K_{a}}{\left(a_{1}-a_{2}\right)}=17.2515 \ldots \ldots \ldots \ldots \ldots \ldots \cdots \cdots \cdots
$$

we can show that matrix $Q_{A C M}$ has two negative eigenvalues $\{-573.27 ;-123.38\}$ with the value $\delta=10^{-4}$. Next, we notice that the ratio $2 R_{a} / R_{c}$ is only $1.9 \times 10^{-4}$ for the front motor and $9.5 \times 10^{-4}$ for the rear motor. In addition, as can be seen in Fig. 9, even if $I_{o d, i}$ reaches $-50[\mathrm{~A}]$ or $+50[\mathrm{~A}], \Psi_{e q, i}$ only deviates $8 \%$ for the front motor, and $1.67 \%$ for the rear motor. This means the practical algorithm in Table 2 is applicable to the three-wheel EV under study.

\section{Test 1: A simple speed pattern}

Fig. 10 describes the setting of Test 1 in which the vehicle has to follow a given speed pattern $v_{r}$ under the change of road friction. In this test, the reference speed $\omega_{r}$ is roughly calculated as $v_{r} / r$. The vehicle operates on the low friction surface from 2 to 4 seconds, and from 25 to 26 seconds.

Test 1-A: Instead of aggregating the motor speeds, we utilize the longitudinal speed measurement $v_{x}$ directly (Fig. 11(a)). The speed response of this test case with the optimal torque-current distribution is shown in Fig. 11(b). Since the speed responses of the front-left and front-right wheels are almost the same, we only plotted the results of the frontleft wheel and rear wheel. The vehicle suffers a large slip of the rear wheel. It consumes an energy of 60.1 [Wh] to cruise a range of $359.2[\mathrm{~m}]$.

Test 1-B: In this case, the control system in Fig. 6 was utilized. The energy consumption with different distribution 


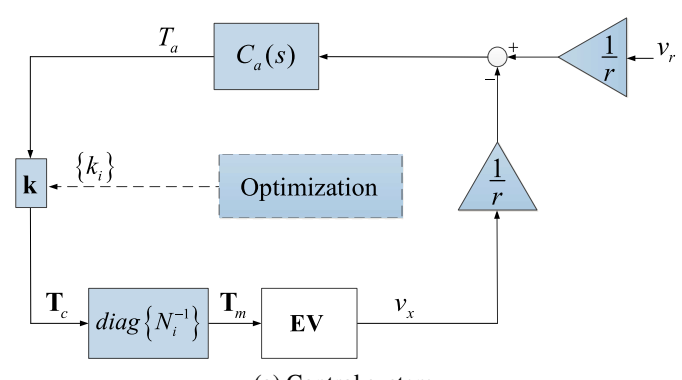

(a) Control system

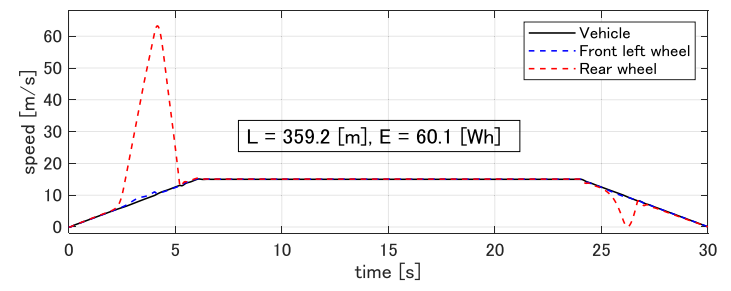

(b) Speed response.

Fig. 11. Test 1-A with vehicle speed sensor

Table 3. Energy consumption of Test 1-B

\begin{tabular}{|c|c|c|}
\hline \multirow{2}{*}{$\begin{array}{c}\text { Torque } \\
\text { distribution }\end{array}$} & \multicolumn{2}{|c|}{ Current distribution } \\
\hline & $I_{o d, i}=0$ & optimal $I_{o d, i}$ \\
\hline \multirow{2}{*}{$\begin{array}{l}\text { Front drives } \\
\left(k_{f}=0.5\right)\end{array}$} & $\mathrm{L}=351.3[\mathrm{~m}]$ & $\mathrm{L}=351.3[\mathrm{~m}]$ \\
\hline & $\mathrm{E}=36.88[\mathrm{Wh}]$ & $\mathrm{E}=32.60[\mathrm{Wh}]$ \\
\hline \multirow{2}{*}{$\begin{array}{c}\text { Rear drives } \\
\left(k_{f}=0.0\right)\end{array}$} & $\mathrm{L}=358.2[\mathrm{~m}]$ & $\mathrm{L}=358.2[\mathrm{~m}]$ \\
\hline & $\mathrm{E}=30.77[\mathrm{Wh}]$ & $E=25.95[\mathrm{Wh}]$ \\
\hline \multirow{2}{*}{$\begin{array}{l}\text { All wheel drives } \\
\qquad\left(k_{f}=1 / 3\right)\end{array}$} & $\mathrm{L}=357.6[\mathrm{~m}]$ & $\mathrm{L}=357.6[\mathrm{~m}]$ \\
\hline & $\mathrm{E}=30.58[\mathrm{Wh}]$ & $\mathrm{E}=26.11[\mathrm{Wh}]$ \\
\hline \multirow{2}{*}{$\begin{array}{c}\text { All wheel drives } \\
\quad\left(k_{f}=0.1\right)\end{array}$} & $\mathrm{L}=357.4[\mathrm{~m}]$ & $\mathrm{L}=357.4[\mathrm{~m}]$ \\
\hline & $27.98[\mathrm{Wh}]$ & $23.28[\mathrm{Wh}]$ \\
\hline \multirow{2}{*}{$\begin{array}{c}\text { All wheel drives } \\
\text { (optimal } k_{f} \text { ) }\end{array}$} & $\mathrm{L}=357.2[\mathrm{~m}]$ & $\mathrm{L}=357.2[\mathrm{~m}]$ \\
\hline & $E=27.53[\mathrm{Wh}]$ & $\mathrm{E}=22.91[\mathrm{Wh}]$ \\
\hline
\end{tabular}

strategies are summarized in Table 3. Even with the worst distribution strategy, energy consumption of Test 1-B is much less than that of Test 1-A. This is because the control system in Test 1-B might prevent the wheel slip to a certain extent. Due to the limitation of paper space, we only presented in Fig. 12 the results of Test 1-B with the optimal torque-current distribution strategy. Again, we only showed the results of the front-left and rear wheels.

For better comparison, the results in Table 3 were normalized by energy consumption per kilometer (Fig. 13). For any torque distribution strategy, $15 \%$ of energy consumption can be saved by optimizing the d-axis current of the motors. In comparison with the front or rear drive strategy, $37.88 \%$ and $25.54 \%$ of energy consumption can be reduced by simultaneously optimizing the torque distribution to all wheels with the optimal d-axis currents.

\section{Test 2: WLTC test}

Test 1 shows that it is possible to aggregate the motor speeds for the cruise control purpose. However, $r \omega_{a}$ is not exactly a representative of the vehicle speed. Fortunately, it is possible to estimate the slip ratio using the motor torque and motor speed ${ }^{(24)}$. Using the definition of slip ratio (7), the vehicle speed can be calculated from the $i$ th motor speed as

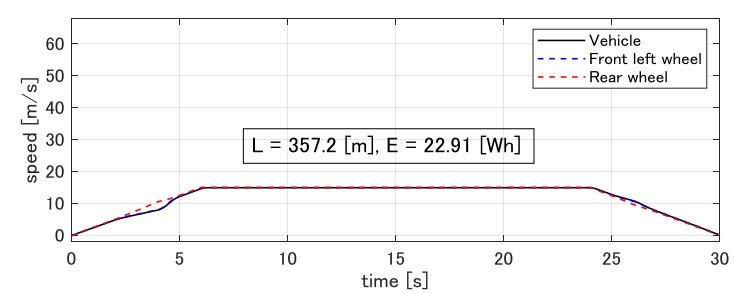

(a) Speed response.

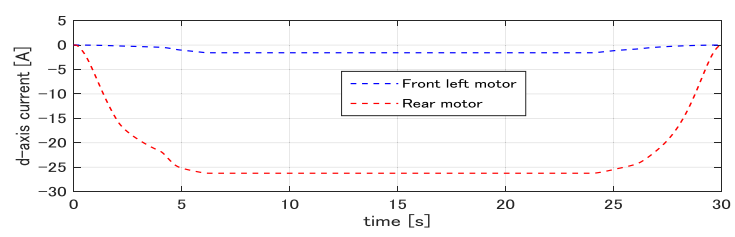

(b) The optimal current $I_{\text {od, } i}$.

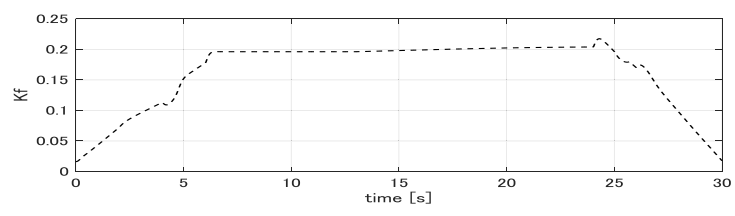

(c) The optimal distribution ratio of the front motors.

Fig. 12. Result of Test 1-B with optimal distribution

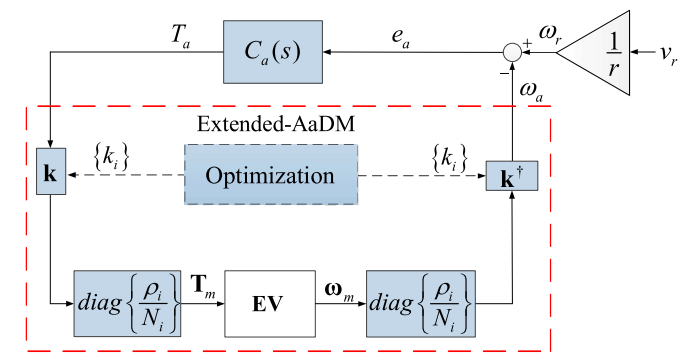

Fig. 14. Extended ACM-RECS for vehicle speed control

$$
\hat{v}_{x, i}=r \rho_{i} N_{i}^{-1} \omega_{m, i}
$$

where

$$
\rho_{i}= \begin{cases}\left(1-\hat{\lambda}_{i}\right) & \text { in acceleration mode } \\ \left(1+\hat{\lambda}_{i}\right)^{-1} & \text { in deceleration mode }\end{cases}
$$

An estimation of the vehicle speed is proposed as follows

$$
\hat{v}_{x}=k_{1} \hat{v}_{x, 1}+k_{2} \hat{v}_{x, 2}+k_{3} \hat{v}_{x, 3}=r \mathbf{k} \operatorname{diag}\left\{\rho_{i} N_{i}^{-1}\right\} \boldsymbol{\omega}_{m}
$$

From (63), an extended version of the ACM-RECS is established in Fig. 14. We did not present the slip ratio estimation algorithms which has been designed and verified ${ }^{(16)(24)}$. Note that the scaling matrix $\operatorname{diag}\left\{\rho_{i} / N_{i}\right\}$ does not change the passivity of the extended AaDM system in the red-dashed rectangle of Fig. 14.

The worldwide harmonized light-duty vehicle test cycle (WLTC) is one of the standard driving-cycles to evaluate the performance of the vehicle. It is used to evaluate the extended ACM-RECS. The test results were summarized in Fig. 15. In this test, the vehicle enters the low friction surface in three periods, $200 \sim 300$ seconds, $700 \sim 800$ seconds, and $1160 \sim 1190$ seconds. Even though, the longitudinal speeds of the wheels are almost close to that of the vehicle body. Similarly to Test 1 , we only demonstrated the results of the 


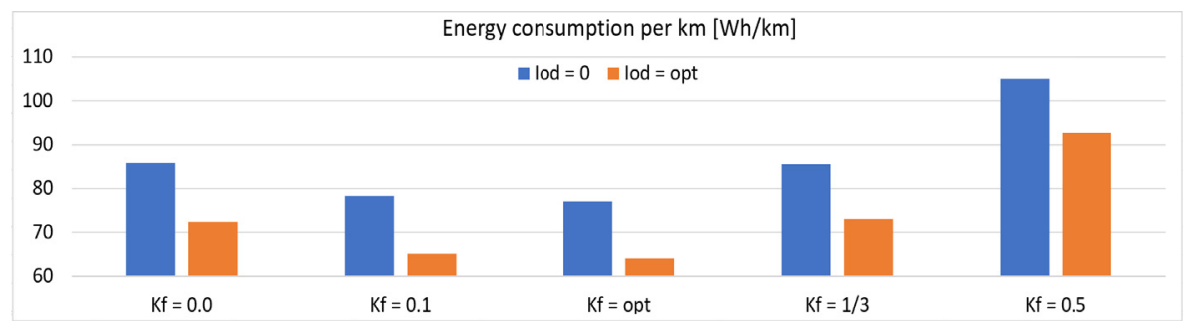

Fig. 13. Comparison of different strategies in Test 1-B

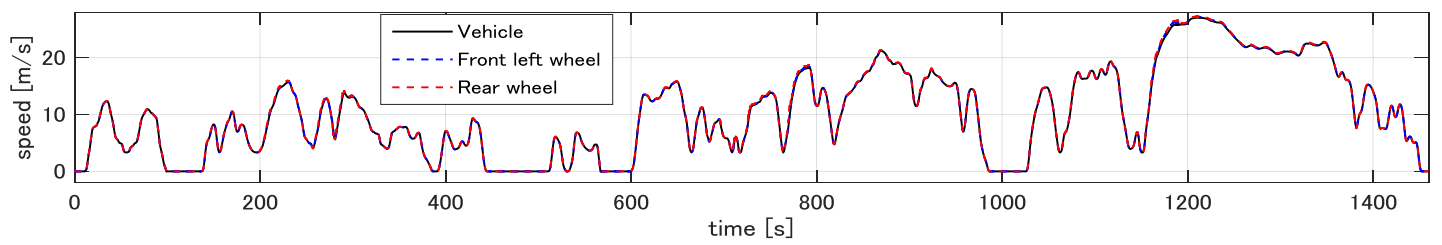

(a) Speed response.

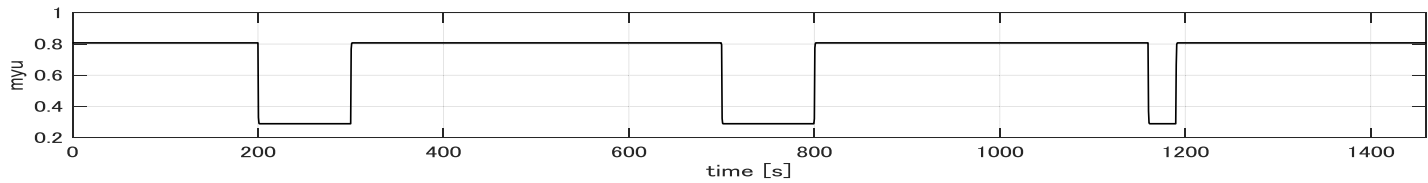

(b) Change of road friction.

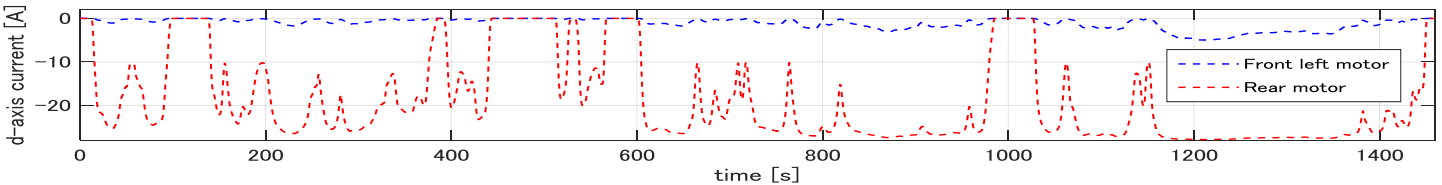

(c) The optimal current $I_{o d, i}$.

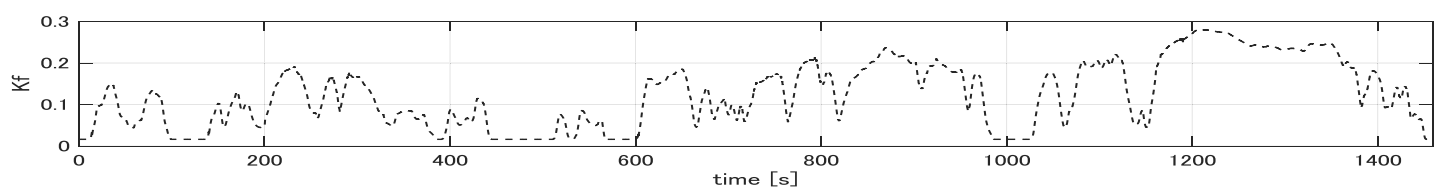

(d) The optimal torque distribution ratio of the front motors.

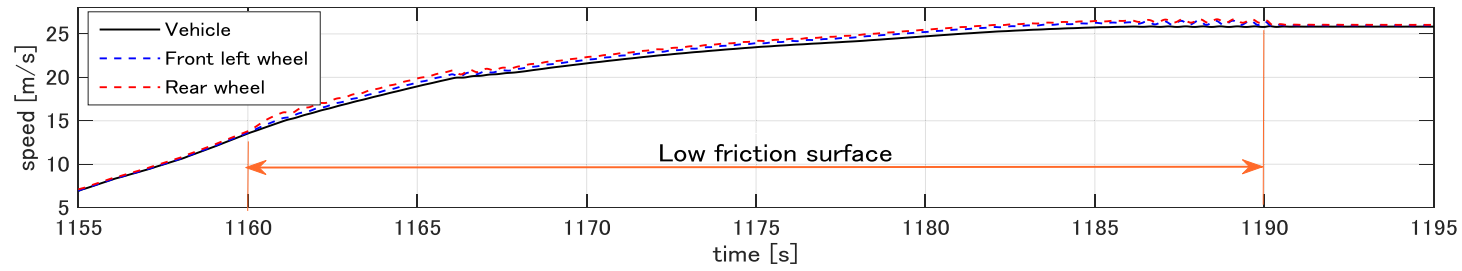

(e) Speed zoom to the most critical period 1160 [s] 1190 [s]: acceleration to the low friction surface with high speed.

Fig. 15. Result of Test 2 using WLTC with optimal torque-current distribution strategy

Table 4. Energy consumption of Test 2

\begin{tabular}{|c|c|c|}
\hline \multirow{2}{*}{$\begin{array}{c}\text { Torque } \\
\text { distribution }\end{array}$} & \multicolumn{2}{|c|}{ Current distribution } \\
\cline { 2 - 3 } & $I_{o d, i}=0$ & optimal $I_{o d, i}$ \\
\hline $\begin{array}{c}\text { All wheel drives } \\
\left(k_{f}=1 / 3\right)\end{array}$ & $\mathrm{E}=1415[\mathrm{Wh}]$ & $\mathrm{E}=1194[\mathrm{Wh}]$ \\
\hline $\begin{array}{c}\text { All wheel drives } \\
\left(k_{f}=0.1\right)\end{array}$ & $\mathrm{E}=1427[\mathrm{Wh}]$ & $\mathrm{E}=1196[\mathrm{Wh}]$ \\
\hline $\begin{array}{c}\text { All wheel drives } \\
\left(\text { optimal } k_{f}\right)\end{array}$ & $\mathrm{E}=1390[\mathrm{Wh}]$ & $\mathrm{E}=1166[\mathrm{Wh}]$ \\
\hline
\end{tabular}

front-left wheel and rear wheel. The energy consumptions in Test 2 are summarized in Table 4. Since the estimated vehicle speed is controlled in this test, the driving range is $14990[\mathrm{~m}]$ for all strategies. In comparison with the worst strategy $\left(k_{f}=0.1\right.$ and $\left.I_{o d, i}=0\right)$, the energy consumption can be reduced $18.29 \%$ by optimizing the torque distribution ratios and d-axis currents.

6.4 Evaluation of HDM-RECS By fine tuning process with the condition (58), we selected a candidate of DOB with the parameters $J_{n}=84.1, D_{n}=0.1, K_{f}=0.8$, $\tau_{f}=0.05$. The HDM-RECS was evaluated by two tests as follows.

Test 3: Short acceleration with sharp change of friction

The setting and results of this test are described in Fig. 16. To accelerates the vehicle, a large driving command $T_{c m d}$ is given by the driver (Fig. 16(a)). As shown in Fig. 16(b), the vehicle enters the low friction surface from 2 to 4 seconds.

In Test 3-A, the DOB is not utilized, which means $T_{a}$ always equals to $T_{c m d}$. As can be seen clearly in Fig. 16(c), 


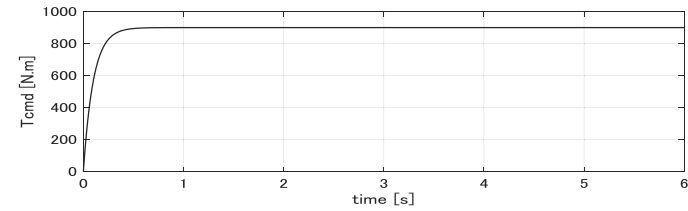

(a) Total driving command.

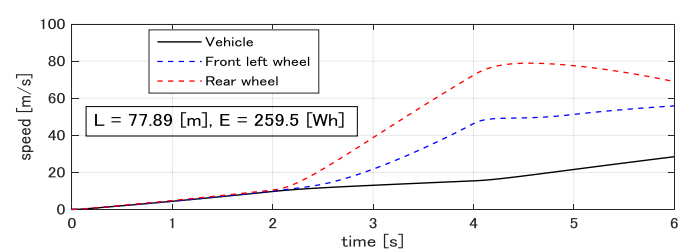

(c) Test 3-A: HDM-RECS without DOB

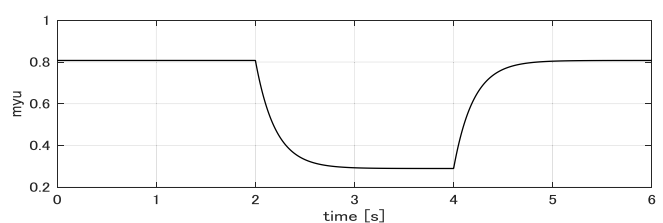

(b) Change of road friction.

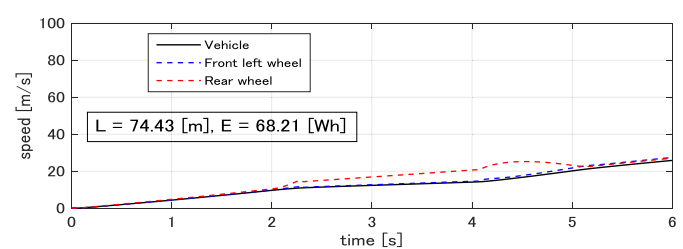

(d) Test 3-B: HDM-RECS with DOB.

Fig. 16. Results of Test 3

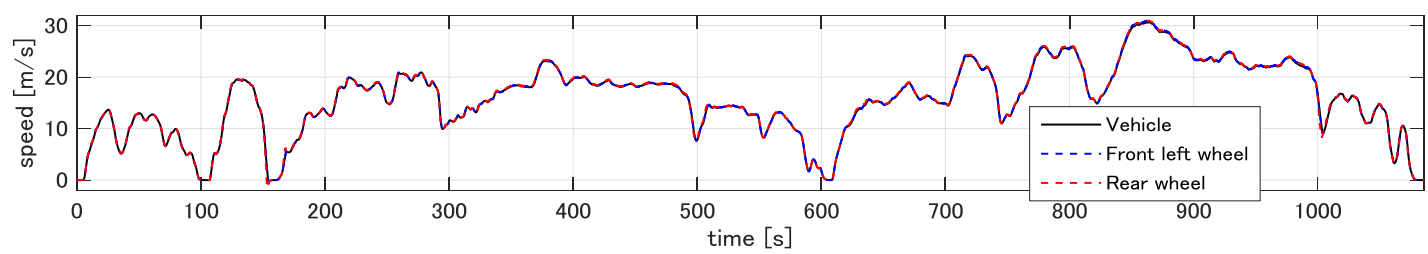

(a) Vehicle speed and wheel speed.

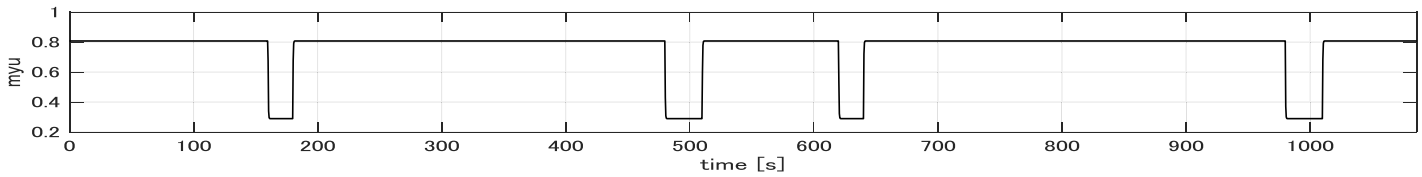

(b) Change of road friction.

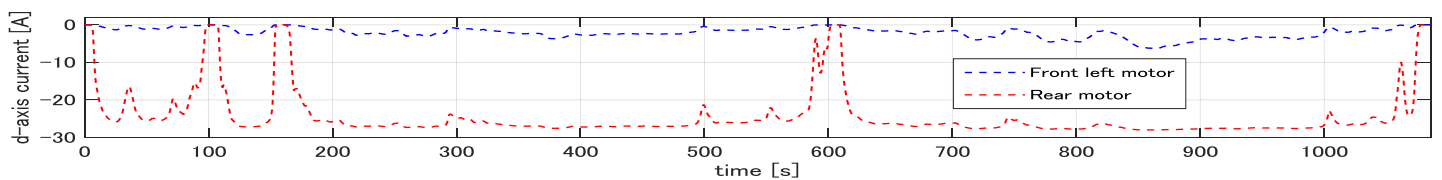

(c) The optimal current $I_{o d, i}$.

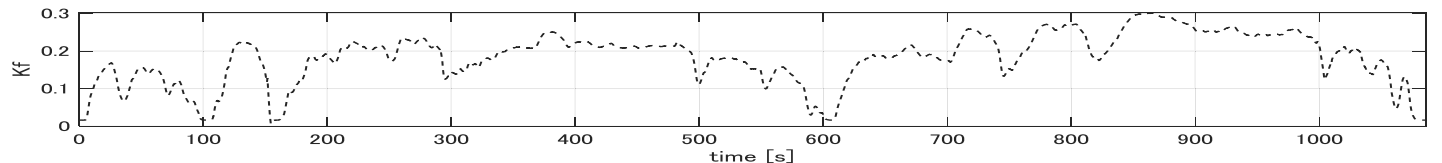

(d) The optimal torque distribution ratio of the front motors.

Fig. 17. Result of Test 4 using CADC with optimal torque-current distribution strategy

the vehicle suffers a noticeable wheel slip phenomenon. Consequently, it requires an energy of 259.5 [Wh] to run a distance of 77.89 [m].

In contrast, Test 3-B utilized the whole control system in Fig. 7. As a result, the wheel slip phenomenon could be prevented to a certain extent (Fig. 16(d)). Certainly, the DOB slightly degrades the acceleration performance in Test 3-B. Therefore, the driving range of Test $3-\mathrm{A}$ is 1.05 times longer than that of Test 3-B. However, the energy consumed by the vehicle in Test 3-A is 3.8 times as much as that of Test 3B. The results showed that proposed control system can improve the safety level and reduce the energy consumption of the RECS.

\section{Test 4: CADC test}

In this test, the driver has to drive the vehicle in the rural schedule of the common Artemis driving cycle (CADC) ${ }^{(25)}$. The setting and results of this test are summarized in Fig. 17. To generate the total driving command $T_{c m d}$, a driver model is established as in the study of Fujimoto et al. ${ }^{(8)}$. Since the
Table 5. Energy consumption of Test 4

\begin{tabular}{|c|c|c|}
\hline \multirow{2}{*}{$\begin{array}{c}\text { Torque } \\
\text { distribution }\end{array}$} & \multicolumn{2}{|c|}{ Current distribution } \\
\cline { 2 - 3 } & $I_{o d, i}=0$ & optimal $I_{o d, i}$ \\
\hline $\begin{array}{c}\text { All wheel drives } \\
\left(k_{f}=1 / 3\right)\end{array}$ & $\mathrm{E}=2017[\mathrm{Wh}]$ & $\mathrm{E}=1710[\mathrm{Wh}]$ \\
\hline $\begin{array}{c}\text { All wheel drives } \\
\left(k_{f}=0.1\right)\end{array}$ & $\mathrm{E}=2041[\mathrm{Wh}]$ & $\mathrm{E}=1721[\mathrm{Wh}]$ \\
\hline $\begin{array}{c}\text { All wheel drives } \\
\left(\text { optimal } k_{f}\right)\end{array}$ & $\mathrm{E}=2000[\mathrm{Wh}]$ & $\mathrm{E}=1690[\mathrm{Wh}]$ \\
\hline
\end{tabular}

DOB is utilized, the longitudinal speeds of the wheels and the vehicle body are almost the same. Similarly to the test with ACM-RECS, we only showed the results in accordance with the front-left wheel and rear wheel.

The energy consumption of Test 4 with several distribution strategies are summarized in Table 5, with the driving 
range of $17150[\mathrm{~m}])$. In comparison with the worst case $\left(k_{f}=0.1\right.$ and $\left.I_{o d, i}=0\right)$, the energy consumption can be reduced $17.20 \%$ by optimizing the torque distribution ratios and the d-axis current.

\section{Conclusions}

This paper shows that to design RECS, energy optimization should be integrated with slip prevention. To this end, this paper introduces the aggregation-and-distribution model (AaDM) which maintains the passivity property of the vehicle. Based on the AaDM, this paper presents an ACMRECS for automatic cruise mode and another HDM-RECS for operating in human driving mode. Using passivity theory, $\mathrm{L}_{2}$ stability conditions and design procedures of two systems were proposed. The condition can be test conveniently via matrix inequality. Under practical assumptions on motor models, this paper derived a practical algorithm for optimizing the torque distribution ratios and d-axis currents of the motors. Although the algorithm only gives an approximated solution of the optimization problem, it can perform analytically, thereby alleviating the computational burden of RECS. The effectiveness of the proposal has been evaluated by various test scenarios using a three-wheel vehicle model. Test results show that, the proposed systems can prevent the slip of the wheel to a certain extent. Besides, given any torque distribution strategy, $15 \%$ of energy consumption can be saved by optimizing the d-axis currents. In comparison with the front drive or rear drive, $37.88 \%$ and $25.54 \%$ of energy consumption can be reduced thanks to the proposed algorithm.

In future study, we are interested in the application of RECS to other EV prototypes, including the vehicle driven by induction motors. We will continue to investigate the optimization problem for the general EV, especially the wellposedness of the problem. Moreover, how to design decentralized DOB while maintaining a simple optimization algorithm, is another interested issue.

\section{Acknowledgment}

We would like to thank Prof. João Pedro F. Trovão, Prof. Minh C. Ta, and Dr. Bao-Huy Nguyên at e-TESC Lab., Department of Electrical and Computer Engineering, University of Sherbrooke, Sherbrooke, QC, J1K 2R1, Canada for their great supports of the electric vehicle model used in this study.

\section{References}

( 1 ) Y. Hori: "Future Vehicle Driven by Electricity and Control-Research on 4 Wheel Motored UOT Mach II", IEEE Transactions on Industrial Electronics, Vol.51, No.5, pp.954-962 (2004)

( 2 ) L. Li, S. Kodama, and Y. Hori: "Design of Anti-Slip Controller for an Electric Vehicle with an Adhesion Status Analyzer Based on the EV Simulator", Asian Journal of Control, Vol.8, No.3, pp.261-267 (2006)

( 3 ) B. Subudhi and S.S. Ge: "Sliding-Mode-Observer-Based Adaptive Slip Ratio Control for Electric and Hybrid Vehicles", IEEE Transactions on Intelligent Transportation Systems, Vol.13, No.4, pp.1617-1626 (2012)

(4) D. Savitski, V. Ivanov, K. Augsburg, T. Emmei, H. Fuse, H. Fujimoto, and L.M. Fridman: "Wheel Slip Control for the Electric Vehicle with In-Wheel Motors: Variable Structure and Sliding Mode Methods", IEEE Transactions on Industrial Electronics, Vol.67, pp.8535-8544 (2020)

( 5 ) J. Amada and H. Fujimoto: "Torque Based Direct Driving Force Control Method with Driving Stiffness Estimation for Electric Vehicle with In-wheelmotor", $38^{\text {th }}$ Annual Conference on IEEE Industrial Electronics Society, pp.4904-4909 (2012)
( 6 ) K. Maeda, H. Fujimoto, and Y. Hori: "Four-wheel Driving-force Distribution Method for Instantaneo us or Split Slipery Roads for Electric Vehicle", Automatika, Vol.54, No.1, pp.103-113 (2013)

( 7 ) S. Harada and H. Fujimoto: "Range Extension Control System for Electric Vehicles Based on Optimal Deceleration Trajectory and Front-Rear DrivingBraking Force Distribution Considering Maximization of Energy Regeneration", $13^{\text {th }}$ International Workshop on Advanced Motion Control, pp.173178 (2014)

( 8 ) H. Fujimoto and S. Harada: "Model-Based Range Extension Control System for Electric Vehicles with Front and Rear Driving-Braking Force Distributions", IEEE Transactions on Industrial Electronics, Vol.62, No.5, pp.32453254 (2015)

( 9 ) Y. Wang, H. Fujimoto, and S. Hara: "Torque Distribution-Based Range Extension Control System for Longitudinal Motion of Electric Vehicles by LTI Modeling with Generalized Frequency Variable", IEEE/ASME Transaction on Mechatronics, Vol.21, No.1, pp.443-452 (2015)

(10) Y. Ikezawa, H. Fujimoto, Y. Hori, D. Kawano, Y. Goto, M. Tsuchimoto, and K. Sato: "Range Extension Autonomous Driving for Electric Vehicles Based on Optimal Velocity Trajectory Generation and Front-Rear DrivingBraking Force Distribution", IEEE Journal of Industry Application, Vol.5, No.3, pp.228-235 (2016)

(11) Y. Suzuki, Y. Kano, and M. Abe: "A Study on Tyre Force Distribution Controls for Full Drive-by-wire Electric Vehicle", Vehicle System Dynamics, Vol.52, pp.235-250 (2014)

(12) C. Chatzikomis, M. Zanchetta, P. Gruber, A. Sorniotti, B. Modic, T. Motaln, L. Blagotinsek, and G. Gotovac: "An Energy-efficient Torque-vectoring Algorithm for Electric Vehicles with Mutiple Motors", Mechanical Systems and Signal Processing, Vol.8, pp.655-673 (2019)

(13) B.-M. Nguyen, M. Kawanishi, S. Hara, H.-V. Nguyen, M. Ta-Cao, J.P. Trovão, and T. Narikiyo: "Passivity Based Hierarchically Decentralized Range Extension Control of In-wheel-motor Vehicles", IEEE International Conference on Mechatronics, pp.1-6 (2021)

(14) S. Morimoto, Y. Tong, Y. Takeda, and T. Hirasa: "Loss Minimization Control of Permanent Magnet Synchronous Motor Drives", IEEE Transactions on Industrial Electronics, Vol.41, No.5, pp.511-517 (1994)

(15) H.B. Pacejka: "Tyre and Vehicle Dynamics", Elsevier BV (2006)

(16) B.-M. Nguyen, S. Hara, H. Fujimoto, and Y. Hori: "Slip Control for IWM Vehicles Based on Hierarchical LQR", Control Engineering Practice, Vol.93, 104179 (2019)

(17) B.-M. Nguyen, H.V. Nguyen, M. Ta-Cao, and M. Kawanishi: "Longitudinal Modelling and Control of In-Wheel-Motor Electric Vehicles as Multi-Agent Systems", Energies, 13, 5437 (2020)

(18) H. Fujimoto, T. Saito, and T. Noguchi: "Motion Stabilization Control of Electric Vehicle Under Snowy Conditions Based on Yaw-Moment Observer", $8^{\text {th }}$ IEEE International Workshop on Advanced Motion Control, pp.35-40 (2004)

(19) R. Bickel and M. Tomizuka: "Passivity Based Versus Disturbance Observer Based Robot Control: Equivalence and Stability”, IFAC Proceedings Volumes, Vol.29, No.1, pp.223-228 (1996)

(20) E. Sariyildiz, H. Sekiguchi, T. Nozaki, B. Ugurlu, and K. Ohnishi: "A Stability Analysis for the Acceleration-Based Robust Position Control of Robot Manipulators via Disturbance Observer", IEEE/ASME Transactions on Mechatronics, Vol.23, No.5, pp.2369-2378 (2018)

(21) H.K. Khalil: "Nonlinear Systems", Prentice Hall (2002)

(22) J.P. Trovao, M.-A. Roux, E. Menard, and M.R. Dubois: "Energy and PowerSplit Management of Dual Energy Storage System for a Three-Wheel Electric Vehicle", IEEE Transactions on Vehicular Technology, Vol.66, No.7, pp.5540-5550 (2016)

(23) A. Van Der Schaft: " $\mathrm{L}_{2}$-Gain and Passivity Techniques in Nonlinear Control", Springer (2017)

(24) K. Fuji and H. Fujimoto: "Traction Control Based on Slip Ratio Estimation Without Detecting Vehicle Speed for Electric Vehicle", 2007 Power Conversion Conference, pp.688-693 (2007)

(25) https://dieselnet.com/standards/cycles/artemis.php 


\section{Appendix}

The main parameters of the three-wheel EV and its figures are listed in app. Table 1.

app. Table 1. Main parameters of the three-wheel EV

\begin{tabular}{|l|l|}
\hline Vehicle mass & $m=430[\mathrm{~kg}]$ \\
\hline Wheel's radius & $r=0.277[\mathrm{~m}]$ \\
\hline Gear transformation ratio & $N_{1}=N_{2}=1, N_{3}=5.033$ \\
\hline Equivalent inertia & $\begin{array}{l}J_{e q, 1}=J_{e q, 2}=0.7807\left[\mathrm{kgm}^{2}\right] \\
J_{e q, 3}=0.1376\left[\mathrm{kgm}^{2}\right]\end{array}$ \\
\hline Cross-sectional area of vehicle in air & $A_{F}=2.37\left[\mathrm{~m}^{2}\right]$ \\
\hline Drag coefficient & $C_{D}=0.35$ \\
\hline Distance from center of gravity to & $L_{f}=0.678[\mathrm{~m}]$ \\
front and rear axles & $L_{r}=1.039[\mathrm{~m}]$ \\
\hline Height of center of gravity & $H_{g}=0.60[\mathrm{~m}]$ \\
\hline Rear motor & $13 \mathrm{~kW}, 120 \mathrm{~N} . \mathrm{m}, p_{n}=10$ \\
\hline Front motor & $4 \mathrm{~kW}, 150 \mathrm{~N} . \mathrm{m}, p_{n}=12$ \\
\hline
\end{tabular}

Binh Minh Nguyen (Member) received his M.S. and Ph.D. degrees

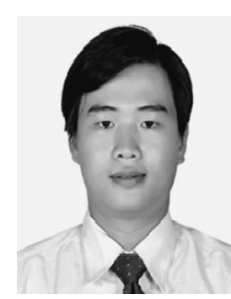
from the University of Tokyo in 2012 and 2015 . He was a researcher at the same university from 2015 to 2020. He is currently a researcher at Toyota Technological Institute. His research interests include glocal control, passivity control, and their applications in electric vehicles, flying vehicles, and power systems. $\mathrm{He}$ is a member of IEEE and IEEJ. Since 2020, he has served as an Associate Editor at the IEEE Vehicular Technology Magazine.

Michihiro Kawanishi (Member) received his M.S. and Ph.D. degrees

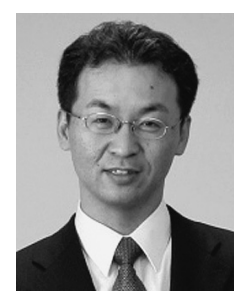
from Kyoto University in 1994 and 1998. He was a Research Associate at Kobe University from 1996 to 2006. In 2006 he became an Associate Professor at Toyota Technological Institute. His research interests include control system design with numerical optimization and its application to mechanical systems. He is a member of IEEE, ISCIE, JSME, and RSJ.
Daichi Hasegawa (Non-member) is currently a student at Toyota

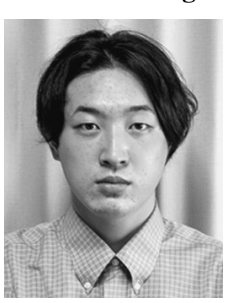
Technological Institute. His research interests include control theory, vehicle dynamics, and multiagent systems.

Kento Ohara (Non-member) is currently a student at Toyota Techno-

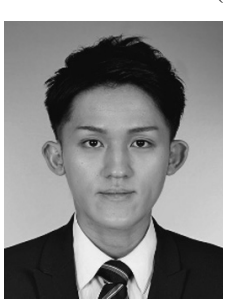
logical Institute. His research interests include passivity, multi-agent system, and electric power systems.

Tatsuo Narikiyo (Non-member) received his B.S., M.S., and Ph.D.

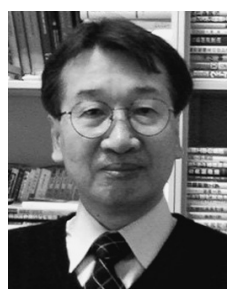
degrees from Nagoya University, Japan, in 1978, 1980, and 1984, respectively. From 1983 to 1990, he was a Research Scientist with the Government Industrial Research Institute. In 1990, he joined the Department of Advanced Science and Technology, Toyota Technological Institute, where he is currently a Distinguished Professor. He retired in 2018. His research interests include nonlinear control systems theory and robotics. 